\title{
Introduction to the Special Issue on Logic Rules and Reasoning: Selected Papers from the 2nd International Joint Conference on Rules and Reasoning (RuleML+RR 2018)
}

\author{
CHRISTOPH BENZMÜLLER \\ Department of Mathematics and Computer Science, Freie Universität Berlin, Berlin, Germany \\ (e-mail: c.benzmueller@fu-berlin.de) \\ XAVIER PARENT \\ Department of Computer Science, University of Luxembourg, Esch-sur-Alzette, Luxembourg \\ (e-mail: x.parent.xavier@gmail.com) \\ FRANCESCO RICCA \\ Department of Mathematics and Computer Science, University of Calabria, Rende, Italy \\ (e-mail: ricca@mat.unical.it)
}

submitted 28 May 2020; accepted 28 May 2020

This volume contains a selection of the best papers of the 2nd International Joint Conference on Rules and Reasoning (RuleML+RR) held from 18 to 21 September 2018 at the Belval campus of the University of Luxembourg. RuleML+RR 2018 was organized as part of the Luxembourg Logic for AI Summit (LuxLogAI 2018), which also included the 4th Global Conference on Artificial Intelligence (GCAI 2018), the DecisionCAMP 2018, the Reasoning Web Summer School (RW 2018), the workshop on Mining and Reasoning with Legal Texts (MIREL 2018), and the annual meeting of the German national interest group in deduction systems (Deduktionstreffen 2018).

RuleML+RR joined the efforts of two well-established conference series: the International Web Rule Symposia (RuleML) and the Web Reasoning and Rule Systems (RR) conferences.

The RuleML symposia and RR conferences have been held since 2002 and 2007, respectively. The RR conferences have been a forum for discussion and dissemination of new results on Web Reasoning and Rule Systems, with an emphasis on rule-based approaches and languages. The RuleML Symposia were devoted to disseminating research, applications, languages, and standards for rule technologies, with attention to both theoretical and practical developments, to challenging new ideas and to industrial applications. Building on the tradition of both RuleML and RR, the joint conference series RuleML+RR aims at connecting academia and industry in the field of rules and to foster the cross-fertilization between the different communities focused on the research, development, and applications of rule-based systems. The RuleML+RR aims at being the leading conference series for all subjects concerning theoretical advances, novel technologies, and innovative applications about knowledge representation and reasoning with rules. 
The technical program of the main track of RuleML+RR 2018 included 25 presentations of peer-reviewed research papers, technical communications, and system demonstrations. As part of the conferences paper selection process, each submission was reviewed by at least three reviewers; many papers additionally received meta-reviews. A selection of four contributions with highest reviewing scores and strong support was identified, and their authors were invited to submit an extended version of their papers to the Journal of Theory and Practice of Logic Programming. After a two-round review process, during which each submission and each revised submission was reviewed by three referees. Three articles were accepted for publication in this special issue.

"Restricted Chase Termination for Existential Rules: a Hierarchical Approach and Experimentation" by Arash Karimi, Heng Zhang, and Jia-Huai You proposes a technique for checking whether a cycle of rule applications might lead to a non-terminating restricted chase on any database by just considering one specific instance. The authors perform an experimental evaluation providing evidence for the efficiency and practical usefulness of the technique for the classes considered in the paper.

"Characterizing Boundedness in Chase Variants" by Stathis Delivorias, Michel Leclère, Marie-Laure Mugnier, and Federico Ulliana studies the termination problem for the chase procedure. In particular, the authors study the boundedness of various chase variants and identify sufficient conditions for decidability of k-boundedness. The importance of $\mathrm{k}$-boundedness of the chase is motivated by the fact that establishing whether the chase terminates on a fact base is undecidable in general. Chase termination is widely recognized as an important problem for the database and logic community, as it is ubiquitous to multiple database and logic-related settings. Indeed, termination of the chase is a desirable property in many applications, since, if the chase terminates, it induces a finite universal model, which can, for instance, be used for answering conjunctive queries or for materializing data in data exchange and integration.

"A Logic-Based Framework Leveraging Neural Networks for Studying the Evolution of Neurological Disorders" by Francesco Calimeri, Francesco Cauteruccio, Luca Cinelli, Aldo Marzullo, Claudio Stamile, Giorgio Terracina, Francoise Durand-Dubief, and Dominique Sappey-Marinier presents an application of logic-based techniques to a realworld problem. The paper introduces an approach to neurological disorder analysis that leverages a combination of machine learning (via neural networks) and answer set programming. The paper outcome is a framework to support physicians and researchers in the diagnosis of the complex mechanisms underlying neurological disorders.

\section{Acknowledgments}

We would like to thank all reviewers for their hard professional work throughout the multi-stage reviewing process. Their observations, comments, and positive criticism led to significant improvements of the accepted papers and they finally enabled the production of this high-quality special issue publication. A special thanks go to Marco Manna, who helped checking a nontrivial technical issue in one of the accepted papers, providing essential input to the final decision process.

Moreover, we are grateful to everybody who helped make RuleML+RR 2018 and LuxLogAI 2018 a success, including the conference chairs, PC members, authors, speakers, and participants. This also includes the sponsors of the events: Binarypark; the 
Department of Computer Science (DCS) at the University of Luxembourg; the Department of Mathematics and Computer Science at the University of Calabria; the Interdisciplinary Centre for Security, Reliability and Trust (SnT) and the Interdisciplinary Lab for Intelligent and Adaptive Systems (ILIAS) at the University of Luxembourg; KPMG Luxembourg; LogicalContracts; the Luxembourg National Research Fund (FNR); oXygen; and Springer.

Finally, we would like to express our thanks and great appreciation to Thomas Eiter, the area editor for Databases and Semantic Web Reasoning of Theory and Practice of Logic Programming, and to Mirek Truszczynski, the editor-in-chief of Theory and Practice of Logic Programming for their continued help and support. 